\title{
Preparation and characterization of methyltrimethoxysilane-Ag nanoparticles using chemical reduction at room temperature
}

\author{
Mahshid Farasat $\cdot$ Mir Maqsood Golzan
}

Received: 31 October 2012/Accepted: 12 March 2013/Published online: 26 March 2013

(C) The Author(s) 2013. This article is published with open access at Springerlink.com

\begin{abstract}
A new, direct, simple and high yield solution phase synthesis for preparing narrow-sized silver nanoparticles by chemical reduction in aqueous solution of $\mathrm{AgNO}_{3}$ in the presence of incorporated Sn particles in methyltrimethoxysilane (MTMS) at room temperature is reported. The resulting MTMS-silver nanoparticles were characterized by means of powder X-ray diffraction and transmission electron microscopy. The produced Ag particles present a tight size distribution with an average diameter of about $15 \mathrm{~nm}$. Dispersed silver nanoparticles in tetrahydrofuran-dimethylformamide $(1: 1)$ mixture exhibit some broad surface plasmon resonance bands in UV-visible spectrum.
\end{abstract}

Keywords Silver nanoparticles · Methyltrimethoxysilane · Chemical reduction $\cdot$ Surface plasmon resonance

\section{Introduction}

Because the recent decades, many studies have been carried out on the materials in the nanoregime particularly on the nanoparticles (NPs) of noble metals. Metal NPs with properties between bulk materials and molecules display interesting linear and nonlinear optical, electronic and chemical properties which differ significantly from those of the corresponding bulk metal (Ozin 1992). Much effort is devoted to research on such systems, because of many potential applications in various fields such as catalysis (Pradhan et al. 2001), surface-enhanced Raman spectroscopy (SERS)

M. Farasat $(\bowtie) \cdot$ M. M. Golzan

Department of Physics, Faculty of Science,

Urmia University, Urmia, Iran

e-mail: farasat62@gmail.com
(Muniz-Miranda et al. 2007), electroanalysis (Welch et al. 2006), development of optical and biological nanosensors, electro-optic materials, laser frequency doubling materials and etc. Among of the various metal NPs, the silver NPs have been widely investigated because of their unusual property exhibition raised due to their size and shape which brings a variety of possibilities with respect to the technical applications. At present, there are lots of techniques available for the synthesis of metal NPs. The procedure for processing nanosized silver particles can briefly be classified into several methods such as: (i) chemical reduction of silver ions (Toshima et al. 1993; Wang et al. 2005), (ii) thermal decomposition in organic solvents (Esumi et al.1990; Sun et al. 2005), (iii) reversed micelle processes (Huang et al. 1996; Egorova et al. 2000), (iv) photoreduction (Jin et al. 2001), (v) ultrasonic radiation (Xiong et al. 2002), (vi) co- $\gamma$ irradiation (Henglein et al. 1999; Temgire et al. 2004), and (vii) microwave irradiation (Pastoriza-Santos et al. 2002). A key issue among these techniques is the particle size-controlling that is very important in applications. Because some of these methods, result in high costs or colloidal productions, most researchers pay more attention to the chemical reduction methods due to their low cost and ease of operating apparatus. Xuping Sun and Yonglan Luo studied the thermal method of Ag NPs synthesis and resulted to colloidal Ag NPs with sizes around 4-24 nm (Sun et al. 2005). Hongshui Wang et al. also synthesized colloidal Ag NPs using chemical reduction method (Wang et al. 2005) and etc.

In this paper, a novel simple and economic method is used for the production of silver nanoparticle through chemical reduction of aqueous solution of $\mathrm{AgNO}_{3}$ in the presence of incorporated metal tin in methyltrimethoxysilane (MTMS) at room temperature. Furthermore, our results show that under study synthetic method have some advantages in comparison with the reported methods such 
as powder produced Ag narrow-sized NPs, size-controlled products and easy produce process.

\section{Experimental}

\section{Material}

Silver nitrate $\left(\mathrm{AgNO}_{3}\right)$, which was purchased from Aldrich, was used without any further purification in this study. Metallic tin powder, tetrahydrofuran (THF), dimethylformamide (DMF) and MTMS $\left(\mathrm{C}_{4} \mathrm{H}_{12} \mathrm{O}_{3} \mathrm{Si}\right.$; MTMS) were also purchased from Merck. Double-distilled water was used during the procedure of the sample preparations.

\section{Procedure of NPs preparation}

Silver NPs were prepared by a typical two-step chemical reduction synthesis method: first, three drops of liquid MTMS were added slowly to $1 \mathrm{~g}$ of metallic tin powder and stirred carefully in which the final specimen should be like as a paste. After stirring the mixture, it was dried at room temperature for an hour to let the $\mathrm{Sn}$ particles remain the MTMS chains. $70 \mathrm{~mL}$ silver nitrate $(1 \mathrm{M})$ was then added gradually to a beaker involving the specimen and was stirred for $10 \mathrm{~min}$. When the dried mixture, which involves Sn particles, was into the aqueous environment, the MTMS forms a nano-porous system inside. So the $\mathrm{AgNO}_{3}$ particles were trapped inside these nano-porous, which contain Sn particles too. The adding silver nitrate process has been repeated for three times with an interval of $6 \mathrm{~h}$ for each time. The final powders were dried at room temperature around $24 \mathrm{~h}$ and were then washed with distilled water for several times until no $\mathrm{NO}_{3}{ }^{-}$ions could be traced. In the presence of these Sn particles, Ag NPs precipitate regarding a chemical process, as follows:

$$
\begin{aligned}
& 2 \mathrm{AgNO}_{3}(\mathrm{~s})+4 \mathrm{H}_{2} \mathrm{O}(1)+\mathrm{Sn}_{2}(\mathrm{~s}) \rightarrow 2 \mathrm{Ag}^{+}+2 \mathrm{NO}_{3}^{-} \\
& +2 \mathrm{H}^{+}+2 \mathrm{OH}^{-}+2 \mathrm{H}_{2} \mathrm{O}+2 \mathrm{Sn}^{+}+2 \mathrm{e}^{-} \rightarrow 2 \mathrm{Ag}(\mathrm{s}) \downarrow \\
& +2 \mathrm{HNO}_{3}+2 \mathrm{Sn}(\mathrm{OH})_{2}
\end{aligned}
$$

In which, $\mathrm{OH}^{-}$radicals will be removed from $\mathrm{Sn}(\mathrm{OH})_{2}$ during the final drying process.

\section{Equipments}

In this paper, the Ag NPs were characterized using Philips CM 200 transmission electron microscopy by placing a drop of the particles in THF on to a carbon film supported on a copper grid. Elemental analysis was obtained using energy dispersive X-ray spectrometer (EDS). The powder $\mathrm{X}$-ray diffraction (XRD) patterns obtained on X'Pert
Philips, using $\mathrm{CuK}_{\alpha}$ radiation, were used for the structural study of the specimens. The absorption measurements were carried out with a Beckman DU 640 spectrometer.

\section{Results and discussion}

XRD study

The crystallinity of silver NPs was assessed from their $\mathrm{X}$-ray powder diffraction patterns. Figure 1 shows the XRD spectra corresponding to Ag NPs prepared (a) with and (b) without MTMS, respectively. As can be seen in Fig. 1 (a), there are three peaks at Bragg angles of 38.10, 44.33 , and 64.42 , respectively. These peaks correspond to the (111) (200), and (220) planes of silver and they indicates silver NPs formation with cubic structure [space group: Fm $3 \mathrm{~m}$ (2 2 5)] with lattice parameter of $a=4.086 \AA$ when they are compared with those data that deduced for the powder diffraction of Ag (File No. 04-0783). Patterns of samples have an overall greater intensity and this could be related to the packing of the powders onto the X-ray plates. A little bit peak shift to low angles in XRD patterns of Ag NPs was observed due to the presence of MTMS (see Fig. 1). Also, the XRD peaks are found to be very broad because of NPs or due to the strain or instrumental errors. The reflections of residual $\mathrm{Sn}$ powder can also be observed on both diffractograms, however, no trace of an $\mathrm{Ag}$ or $\mathrm{Sn}$ oxide phases were detected. The particle sizes of both samples were calculated by substituting full width at half maximum (FWHM) and position of intense peak in Scherrer's equation:

$D=\frac{0.9 \lambda}{\beta \cos \theta}$

where $D$ corresponds to the particle size in $\AA, \lambda$ is the $\mathrm{X}$-ray wavelength equal to $1.54 \mathrm{~nm}, \beta$ corresponds to FWHM in radian and $\theta$ is the half diffraction angle. By considering Eq. (1), it is found that the crystallite diameter of synthesized Ag NPs in the presence of MTMS $(19.12 \mathrm{~nm})$ is smaller than those that synthesized in the absence of it $(40.43 \mathrm{~nm})$. The particle size differences were approximately $20 \mathrm{~nm}$.

\section{TEM study}

Figure 2 shows the TEM images of chemical reduction of Ag NPs in the presence of MTMS at room temperature (Fig. 2a) after 3 months storage (Fig. 2b) and after 6 months storage (Fig. 2c) at room temperature. It can be seen that the shape of the particles are spherical. Figure $2 b$ shows TEM image of storage Ag NPs after 3 months. As can be seen in Fig. 2b Ag NPs started to aggregate locally. 
Fig. 1 XRD diffractograms of prepared Ag nanoparticles (A) with MTMS $(B)$ without MTMS

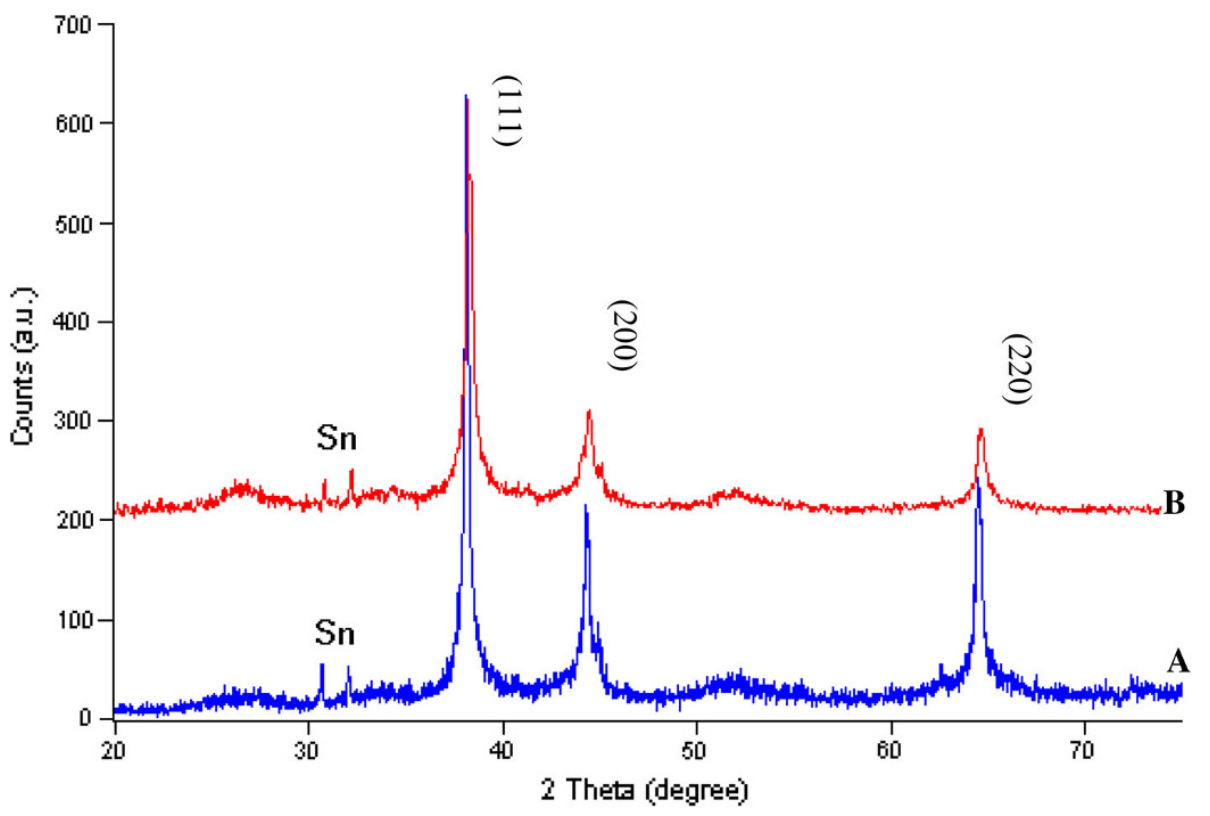

More aggregated, NPs have been appeared after 6 months (see Fig. 2c). The results also indicate that increasing of $\mathrm{Ag}$ NP diameters has been started from third month. Analyzing a hundred of particles from a portion of the grid indicates that the average diameter of the particles is around $15 \mathrm{~nm}$ (see Fig. 3).

Figure 4 shows the EDS spectrum of randomly selected Ag NPs. As shown, the dominating signal comes from the silver. The signals of carbon and copper also belong to the used net holding material. Negligible quantity of $\mathrm{Sn}$ and $\mathrm{Si}$ are also visible. Si peak indicates that Ag NPs form in MTMS matrix.

\section{UV-visible study}

Expectedly, as prepared Ag NPs in the presence of MTMS in THF-DMF (1:1) mixture exhibit some broad surface plasmon resonance (SPR) bands in UV-visible spectrum (see Fig. 5). Figure also shows the decomposition of absorption bands into individual multipole contributions sub-bands. In addition, Fig. 5 clearly reveals that the maxima in the absorption spectrum, which are caused by a strong enhancement of higher multipoles in the case of evanescent-wave excitation. The electric quadrupole and particularly the electric octupole appear in the range of shorter wavelengths and many peaks structures due to electric dipoles SPR appear at longer wavelengths. It is worth to note that these peaks could not originate from light interference or from impurities in the sample.

\section{Comparative study}

In this paper, we succeed to achieve a method of Ag NPs synthesizing with mean particle sizes from 15 to $24 \mathrm{~nm}$. To control Ag NP sizes, different amount of metallic tin powder and $\mathrm{AgNO}_{3}$ have been used. The results showed particle sizes increased with decreasing of metallic Sn and liquid $\mathrm{AgNO}_{3}$, where the diameter of synthesized Ag NPs more affected by Sn fluctuation (see Table 1).

Several methods have been used to synthesize silver NPs with well-defined shapes. A number of these methods along with our results are briefly listed in Table 2. By comparing them, it seems that our suggested procedure is a new, simple and economic method and has the potential to obtain silver NPs with well-defined morphologies. Maybe the use of some processes and methods like gas and ultrasonic aided reductions could produce very small particles with size of less than $10 \mathrm{~nm}$ and narrow grain size distribution, but the expensive materials and equipments result in high cost, consequently are not economical. In addition, the present work has done at room temperature without any further unnecessary conditions or equipments. In addition, boiling and refluxing were not used as the heat-induced process in the shape evolution of silver NPs (Wang et al. 2005; Yu et al. 2004; Gervais et al. 2001; Balan et al. 2007; Iglesias-Silva et al. 2007; Min et al. 2006; Yin et al. 2004). Based on our best results, it also seems that MTMS acts as a binder and/or size controller material. 

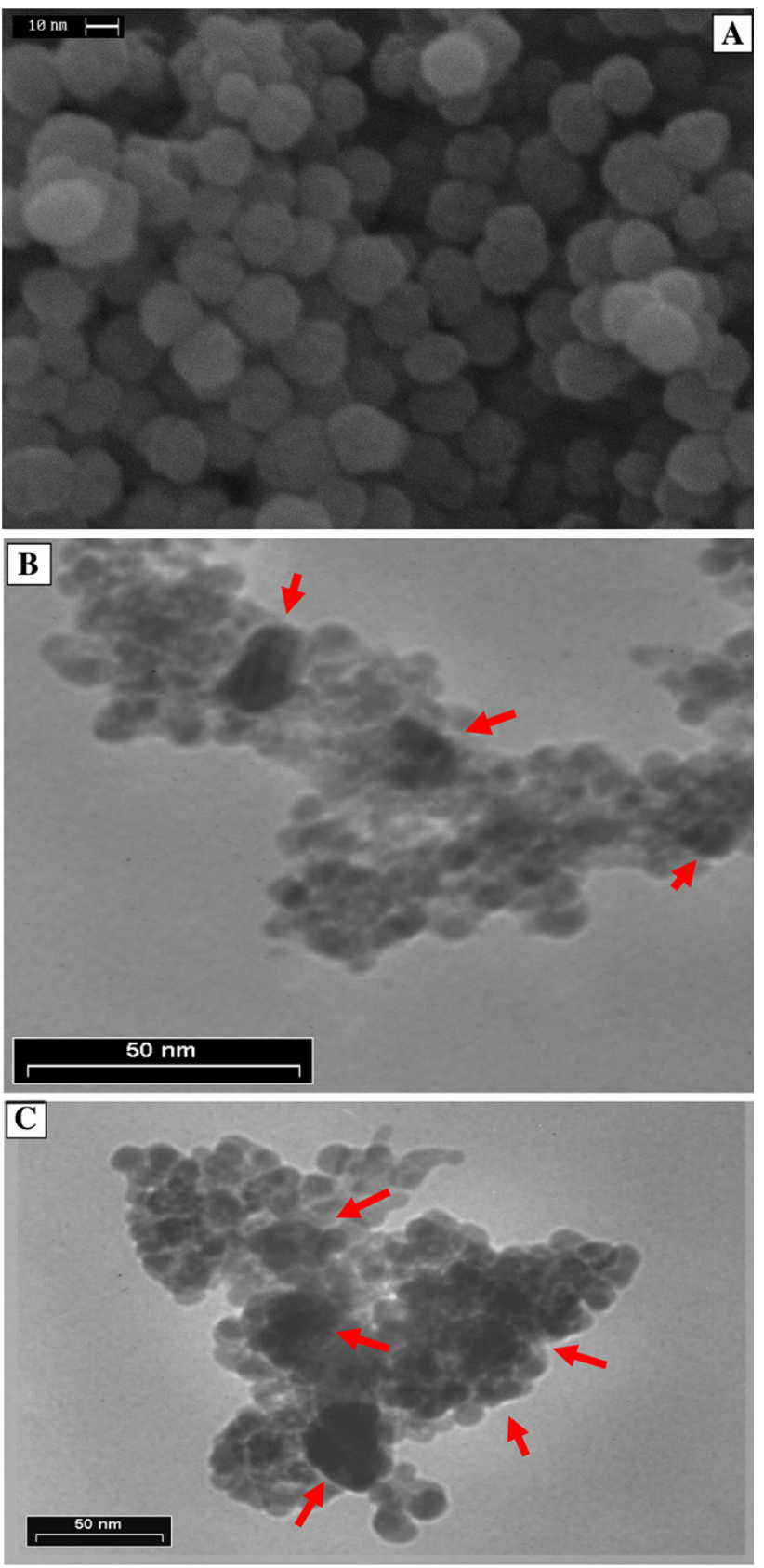

Fig. 2 TEM image of as prepared Ag nanoparticles in the presence of MTMS (a), after 3 months storage (b) and after 6 months storage (c) at room temperature

\section{Conclusion}

Ag NPs were prepared by a simple solution phase chemical reduction reaction of $\mathrm{AgNO}_{3}$ using metallic tin powder in the presence of MTMS in water at room temperature. Based on our proposed method, nearly spherical shape particles can be obtained and particle sizes were the under the control of MTMS. Prepared Ag NPs show some broad SPR bands in UV-visible region which have very important role from the industrial and theoretical points of view.

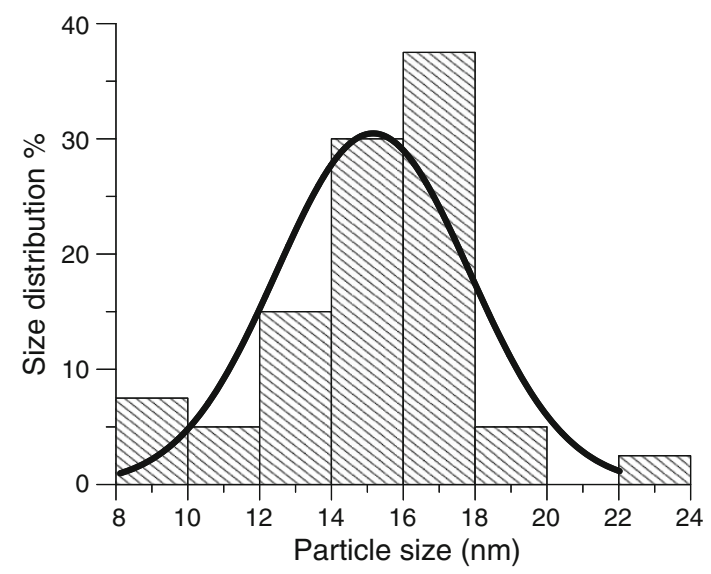

Fig. 3 Particle sizes analysis histogram of samples calculated from TEM image



Fig. 4 EDS analysis of prepared Ag nanoparticles in the presence of MTMS



Fig. 5 UV-visible absorption spectrum of prepared $\mathrm{Ag}$ nanoparticles in the presence of MTMS in THF-DMF (1:1) mixture along with decomposed sub-bands 
Table 1 Experimental parameters of samples

\begin{tabular}{llll}
\hline Sample & Liquid $\mathrm{AgNO}_{3}(\mathrm{M})$ & Metallic tin powder $(\mathrm{gr})$ & Mean particle size $(\mathrm{nm})$ \\
\hline Present work & 1 & 1 & 15.14 \\
$\mathrm{~A}_{1}$ & 1 & 0.22 & 23.86 \\
$\mathrm{~A}_{2}$ & 0.01 & 1 & 15.75 \\
\hline
\end{tabular}

Table 2 A comparison between reported Ag nanoparticle synthesis methods and present work results

\begin{tabular}{|c|c|c|c|c|c|c|c|}
\hline Method & Initial reagent & Reducing material & Solvent & Stabilizer or binder & Condition & $\begin{array}{l}\text { Particle } \\
\text { sizes }(\mathrm{nm})\end{array}$ & Reference \\
\hline $\begin{array}{l}\text { Chemical } \\
\text { reduction }\end{array}$ & $\mathrm{AgNO}_{3}$ & Sn & Water & MTMS & $\begin{array}{l}\text { Room } \\
\text { temperature }\end{array}$ & $15-24$ & Present work \\
\hline $\begin{array}{l}\text { Solution phase } \\
\text { synthetic route }\end{array}$ & $\mathrm{AgNO}_{3}$ & $\mathrm{NaH}$ & THF & $\mathrm{t}-\mathrm{BuONa}$ & $\begin{array}{l}\text { Low } \\
\text { temperature }\end{array}$ & $3.3 \pm 0.7$ & $\begin{array}{l}\text { (Balan et al. } \\
\text { 2007) }\end{array}$ \\
\hline $\begin{array}{l}\text { Hydrothermal } \\
\text { process }\end{array}$ & $\mathrm{Ag}\left(\mathrm{NH}_{3}\right)_{2} \mathrm{NO}_{3}$ & $\begin{array}{l}\text { Poly( } N \text {-vinyl-2- } \\
\text { pyrrolidone) }\end{array}$ & Water & - & $\begin{array}{l}\text { Hydrothermal } \\
\text { treatment }\end{array}$ & $13 \pm 4$ & $\begin{array}{l}\text { (Iglesias-Silva } \\
\text { et al. 2007) }\end{array}$ \\
\hline $\begin{array}{l}\text { Chemical } \\
\text { reduction }\end{array}$ & $\mathrm{AgNO}_{3}$ & $\begin{array}{l}\text { PVP, glucose and } \\
\text { sodium hydroxide }\end{array}$ & Distilled water & - & $\begin{array}{l}\text { Temperature } \\
60{ }^{\circ} \mathrm{C}\end{array}$ & $20-80$ & $\begin{array}{l}\text { (Wang et al. } \\
\text { 2005) }\end{array}$ \\
\hline $\begin{array}{l}\text { Gamma- } \\
\text { radiation }\end{array}$ & $\mathrm{AgNO}_{3}$ & ${ }^{60} \mathrm{Co}$-gamma-ray & $\begin{array}{c}\text { Ethylene } \\
\text { glycol }\end{array}$ & $\begin{array}{l}\text { 4-vinylpyridine or } \\
\text { poly(4- } \\
\text { vinylpyridine) }\end{array}$ & $\begin{array}{l}\text { Under } \\
\text { Ambient } \\
\text { condition }\end{array}$ & $5-20$ & $\begin{array}{l}\text { (Yu et al. } \\
2004)\end{array}$ \\
\hline $\begin{array}{l}\text { Ultrasonic } \\
\text { Radiation }\end{array}$ & $\mathrm{AgNO}_{3}$ & Nano-carbon & $\begin{array}{l}\text { Water and } \\
\text { alcohol }\end{array}$ & - & $350-500$ & 20.4 & $\begin{array}{l}\text { (Min } \\
\text { et al.2006) }\end{array}$ \\
\hline $\begin{array}{l}\text { Microwave } \\
\text { irradiation }\end{array}$ & $\mathrm{AgNO}_{3}$ & Formaldehyde & $\begin{array}{l}\text { Ion-exchanged } \\
\text { water }\end{array}$ & Trisodium citrate & - & $24-132$ & $\begin{array}{l}\text { (Yin et al. } \\
2004)\end{array}$ \\
\hline
\end{tabular}

Open Access This article is distributed under the terms of the Creative Commons Attribution License which permits any use, distribution, and reproduction in any medium, provided the original author(s) and the source are credited.

\section{References}

Balan L, Malva J-P, Schneider R et al (2007) Preparation and properties of poly(urea-formaldehyde) microcapsules filled with epoxy resins. Mater Chem Phys 104:417-425

Egorova EM, Revina AA (2000) Synthesis of metallic nanoparticles in reverse micelles in the presence of quercetin. Colloids Surf A 168:87-96

Esumi K, Tano T et al (1990) Preparation and characterization of bimetallic palladium-copper colloids by thermal decomposition of their acetate compounds in organic solvents. Chem Mater 2(5):564-567

Gervais C, Smith ME, Pottier A et al (2001) Solid-state 47,49Ti NMR determination of the phase distribution of titania nanoparticles. Chem Mater 13:462-467

Henglein A, Giersig M (1999) Formation of colloidal silver nanoparticles: capping action of citrate. Phys Chem B 103:9533-9539

Huang HH, Ni XP et al (1996) Photochemical formation of silver nanoparticles in poly ( $N$-vinylpyrrolidone). Langmuir 12:909-912

Iglesias-Silva E, Rivas J, Leo'n Isidro LM et al (2007) Synthesis of silver-coated magnetite nanoparticles. J Non-Cryst Solids 353:829-831

Jin $\mathrm{R}$ et al (2001) Photoinduced conversion of silver nanospheres to nanoprisms. Science 294:1901-1903

Min Z, Zuo-shan W, Ya-wei Z (2006) Trans. Preparation of silver nanoparticle via active template under ultrasonic 16:1348-1352
Muniz-Miranda M, Pergolese B, Bigotto A et al (2007) A Raman and AFM investigation on metal surfaces SERS-activated by silver colloidal nanoparticles. Mater Sci Eng C 27:1295-1299

Ozin GA (1992) Nanochemistry: synthesis in diminishing dimensions. Adv Mater 4:612-649. doi:10.1002/adma.19920041003

Pastoriza-Santos I, Liz-Marzan LM (2002) Formation of PVPProtected Metal Nanoparticles in DMF. Langmuir 18:2888-2894

Pradhan N, Pal A, Pal T (2001) Catalytic reduction of aromatic nitro compounds by coinage metal nanoparticles. Langmuir 17:1800 1802

Sun X, Luo Y (2005) Preparation and size control of silver nanoparticles by a thermal method. Mater Lett 59:3847-3850

Temgire K, Joshi SS (2004) Optical and structural studies of silver nanoparticles. Radiat Phys Chem 71:1039-1044

Toshima N, Yonezawa T, Kushihashi K (1993) Polymer-protected palladium-platinum bimetallic clusters: preparation, catalytic properties and structural considerations. J Chem Soc Faraday Trans 89:2537-2543

Wang H, Qiao X, Chen J et al (2005) Preparation of silver nanoparticles by chemical reduction method. Coll Surf A Physicochem Eng Aspects 256:111-115

Welch CM, Compton RG (2006) The use of nanoparticles in electroanalysis: a review. Anal Bioanal Chem 384:601-619

Xiong X, Xie Y, Du G et al (2002) Ultrasound-assisted self-regulation route to Ag nanorods. Chem Lett 31:98-99

Yin H, Yamamoto T, Wada Y et al (2004) Large-scale and sizecontrolled synthesis of silver nanoparticles under microwave irradiation. Mater Chem Phys 83:66-70

$\mathrm{Yu}$ D, Sun X, Bian J et al (2004) Gamma-radiation synthesis, characterization and nonlinear optical properties of highly stable colloidal silver nanoparticles in suspensions. Physica E 23:50-55 